UCRL-ID-139032

\title{
Use of Imploding Spheres: an Alternative to Explosives as Acoustic Sources at Mid-Latitude SOFAR Channel Depths
}

\author{
P. E. Harben, C. Boro, L. Dorman and J. Pulli
}

\section{May 12, 2000}




\section{DISCLAIMER}

This document was prepared as an account of work sponsored by an agency of the United States Government. Neither the United States Government nor the University of California nor any of their employees, makes any warranty, express or implied, or assumes any legal liability or responsibility for the accuracy, completeness, or usefulness of any information, apparatus, product, or process disclosed, or represents that its use would not infringe privately owned rights. Reference herein to any specific commercial product, process, or service by trade name, trademark, manufacturer, or otherwise, does not necessarily constitute or imply its endorsement, recommendation, or favoring by the United States Government or the University of California. The views and opinions of authors expressed herein do not necessarily state or reflect those of the United States Government or the University of California, and shall not be used for advertising or product endorsement purposes.

Work performed under the auspices of the U. S. Department of Energy by the University of California Lawrence Livermore National Laboratory under Contract W-7405-Eng-48.

This report has been reproduced

directly from the best available copy.

Available to DOE and DOE contractors from the

Office of Scientific and Technical Information

P.O. Box 62, Oak Ridge, TN 37831

Prices available from (423) 576-8401

http:/ /apollo.osti.gov/bridge/

Available to the public from the

National Technical Information Service

U.S. Department of Commerce

5285 Port Royal Rd.,

Springfield, VA 22161

http://www.ntis.gov/

OR

Lawrence Livermore National Laboratory

Technical Information Department's Digital Library

http://www.llnl.gov/tid/Library.html 


\begin{abstract}
$\underline{\text { Abstract }}$
The hydroacoustic nuclear explosion monitoring regime, like it's counterpart in seismic monitoring, requires ground truth calibration. Model predictions of traveltimes, blockages, reflections, diffractions, and waveform envelopes need to be verified with ground truth experiments, particularly in the high latitudes where models often fail. Although pressure detonated explosives are a simple, reliable, and flexible method to generate an impulsive hydroacoustic calibration source at a desired depth; safety procedures, specialized training, and local regulations often preclude their use. This leaves few alternatives since airgun and other seismic marine sources are designed for use only at shallow depths and hence do not effectively couple into the SOFAR channel, a necessary requirement for long range propagation. Imploding spheres could be an effective source at mid-ocean depths and below but development of a method to reliably break such spheres has been elusive.
\end{abstract}

We designed and tested a prototype system to initiate catastrophic glass sphere failure at a prescribed depth. The system firmly holds a glass sphere in contact with a piston-ram assembly. The end cap on the cylinder confining the piston and opposing the ram has a rupture disk sealed to it. The rupture disk is calibrated to fail within $5 \%$ of the calibrated failure pressure, 1000 psi in our tests. Failure of the rupture disk results in a sudden inrush of high pressure water into the air-filled piston chamber, driving the piston -- and attached ram -- towards the glass sphere.

The spherecracker was first tested on Benthos Corp. flotation spheres. The spherecracker mechanism successfully punched a hole in the Benthos sphere at the nominal pressure of 1000 psi or at about 700 meters depth in each of four tests. Despite the violent inrush of high pressure water the spheres did not otherwise fail. We concluded that the Benthos spheres were too thick-walled to be used as an imploding source at nominal SOFAR channel depths (500 meters - 1200 meters in midlatitudes). A much thinner walled sphere, a special order modification of a standard 22 liter laboratory boiling flask made by the Kontes Glass Company, was also tested and found to fail in the desired manner, i.e. catastrophically. A test off the coast of California successfully initiated implosion of a Kontes sphere at 685 meters depth. The recorded signal showed a peak pressure slightly larger than that from $1 \mathrm{lb}$ of high explosive detonated at the same depth. The signal spectra showed relatively broad band higher frequency energy with little signal below about $50 \mathrm{~Hz}$ and a broad peak in the amplitude spectra between about 200 and $800 \mathrm{~Hz}$, similar to that from an explosive source detonated at the same depth.

Although additional testing and development is needed, an imploding sphere source for hydroacoustic calibrations appears viable. Since the source spectra frequencies are generally higher than the frequency band used for nuclear explosion monitoring, low frequency signals $(1-50 \mathrm{~Hz})$ will be absent from the implosion source spectra. Calibration will have to be accomplished with frequencies above $50 \mathrm{~Hz}$ unless larger spheres, multiple spheres, or shallower implosion depths are used. 


\section{Introduction}

The hydroacoustic monitoring regime for the Comprehensive Nuclear-Test-Ban Treaty, like it's counterpart in seismic monitoring, requires ground truth calibration. Although the relative homogeneity of the ocean medium compared to the solid earth has allowed for more accurate and reliable computer models of acoustic propagation, significant problems and errors in the acoustic propagation models remain. Model predictions of traveltimes, blockages, reflections, diffractions, and waveform envelopes need to be verified with ground truth experiments on as many paths as possible if hydroacoustic monitoring capabilities are to be enhanced. One way to accomplish such ocean basin calibrations is to detonate a large number of small ( $1-2 \mathrm{lbs}$ of high explosive) charges within the SOFAR axis (600 meters to 1200 meters nominal depth) on various tracks in an ocean basin, recording the signals at all operational hydroacoustic stations.

Although pressure-detonated explosives are a simple, reliable, and flexible method to generate an impulsive hydroacoustic source at a desired depth; safety procedures, specialized training, and local regulations preclude their use on most ships. This leaves few alternatives as SOFAR axis sources, since airgun and most other seismic marine sources are designed for use only at shallow depths. Imploding spheres have a relatively long history of specialized use and are a potential acoustic source at mid-ocean depths and below; but they have not been deemed reliable because they fail well below or above the desired depth or do not fail catastrophically at all. If imploding spheres can be made reliable and can deliver an adequate signal for long range calibration, the method has the distinct advantage of having no stored energy at the surface and therefore no shipboard safety concerns and no special shipboard equipment requirements for deployment. This advantage allows for the possibility of deploying the spheres from a wide variety of ships on cruises for other purposes, thereby minimizing the costs associated with ground truth data collection. The need for an inexpensive source at the SOFAR axis that does not have the hazard and safety procedure problems of explosives was recognized in the recent International Workshop on Hydroacoustic Monitoring for the CTBT that met in Tahiti late September, 1999 [1]. The workshop findings identify acoustic source development as a technical issue and advocate an approach to the problem that addresses the safety issue and the need for low cost expendable sources.

Implosion sources have been used in specialized oceanographic studies for nearly 50 years. In 1952, 0.01 liter glass floats were imploded as an acoustic signal for indicating that a sediment corer reached the ocean bottom [2]. In 1976, a study used weakened glass spheres to vary the depth of implosion [3]. Most recently (1996), a project to develop a deep water ocean bottom implosive source using a 20 liter cylinder was conducted [4]. The deep water source initiated cylinder implosion with a pyrotechnic igniter device. We do not know of an existing implosion system suited to the hydroacoustic monitoring needs. Any development of such a system must show utility by demonstrating good source properties (amplitude and frequency content), implosion depth control, reliability, repeatability, and long range propagation.

This paper will describe a sphere implosion system and the results of early field tests. This is a report of work-in-progress, serving to document some preliminary experiments and is not a complete study. First, we will qualitatively examine the properties of an imploding acoustic source. We will then outline the design of an implosion initiation device, or spherecracker, for causing glass sphere implosions at predetermined depths without the use of explosives or stored energy 
devices. This will be followed by an outline of early field tests culminating in the most recent successful test that recorded the source waveform with a calibrated hydrophone. Finally, we will conclude by outlining future tests and research issues that need to be completed before using this type of source. Potential applications for hydroacoustic calibration for nuclear explosion monitoring will also be discussed.

\section{Acoustic properties of imploding spheres}

An imploding source exploits the pressure difference between an enclosed volume of gas at nominal atmospheric pressure and the external water pressure at the implosion depth. A sudden catastrophic failure of the containing vessel leaves the relatively low pressure gas bubble exposed to relatively high pressure water and a rapid implosion ensues. The implosion momentum collapses the bubble radius to less than that required for an equilibrium pressure balance. At the instant of minimum bubble radius, the bubble begins expanding and radiates a positive acoustic pressure spike. This oscillation can continue for a few cycles, each with successively reduced pressure spikes as energy is dissipated and the bubble approaches a static equilibrium pressure. The process is similar to the bubble pulses seen from subsurface explosions; the gas globe from the explosion oscillating about the equilibrium pressure. One distinct difference that in the case of underwater explosions there is an initial shock wave caused by ignition of the explosive and creation of the expanding gas globe consisting of explosion gas products. There is no such analog in an implosion. Another important difference is the relatively cold low pressure gas inside the sphere compared to the high temperature dense explosive gas products in the gas globe. The bubble collapse details of the two cases cannot be directly compared.

At nominal SOFAR depths $(500 \mathrm{~m}$ to $1000 \mathrm{~m}$ ), the energy density spectrum of a $1 \mathrm{lb}$ TNT explosion is broadband with a peak amplitude between 150 and $300 \mathrm{~Hz}$. As will be shown, the spectrum of an imploding sphere is also broadband, but the peak amplitude is slightly higher in frequency and even broader. Higher frequency acoustic energy has implications for long range propagation since acoustic attenuation in seawater increases with increasing frequency. Furthermore, the relative lack of low frequency in the source (i.e. $1-80 \mathrm{~Hz}$ ) -- the frequency band that is monitored by CTBT hydroacoustic sensors -- puts some limitations on the utility of the source for some aspects of calibration. This will be discussed in greater detail in the following sections.

If a glass sphere is of sufficient volume to produce the desired source signal level and has sufficient wall thickness to survive the water pressures in the operational depth range, the sphere failure must be initiated by some controlled method at a predetermined depth. Such a mechanical implosion initiation device was designed to crack the sphere at a preset depth, herein referred to as the "spherecracker".

\section{Design of a failure initiation device}

The failure initiation device, or spherecracker, was designed to be rugged enough to be reusable, heavy enough to sink the whole assembly loaded with the sphere, and without stored energy at the surface. In addition, use of inexpensive materials from standard stock was also a goal so that the spherecracker cost is low enough to allow for a use-once option in deployment. The device is shown in Figure 1 and consists of two orthogonal cut-out plates that hold the sphere and a cylin- 
der-piston-ram assembly that punches a hole in the sphere. The system firmly holds the sphere in place and in contact with a 4 inch diameter piston. A $1 / 4$ inch diameter ram is connected to the center of the piston and passes through a small O-ring sealed hole in the cap confining the piston and abutting the glass sphere. The ram initiates failure by punching a hole through the glass sphere. The end-cap on the cylinder confining the piston and opposing the ram end-cap tapers to a one inch diameter opening with a rupture disk sealed to it. The rupture disk is calibrated to fail within $5 \%$ of the calibrated failure pressure, $1000 \mathrm{psi}$ in our tests. Failure of the rupture disk results in an inrush of high pressure water into the air-filled piston chamber, driving the piston -and attached ram -- towards the glass sphere.

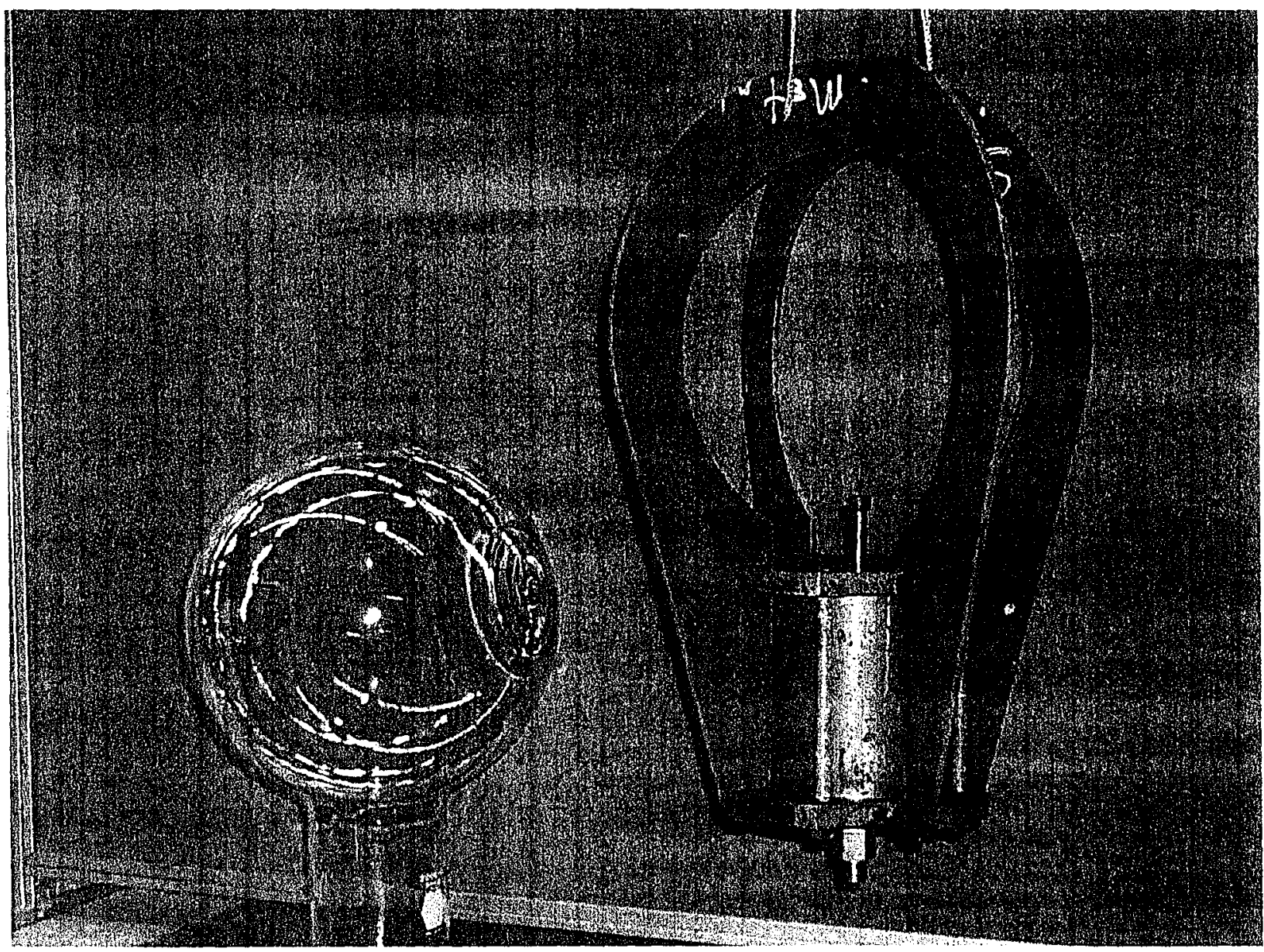

Figure 1) The spherecracker is shown on the right in the lowering orientation (as opposed to the cylinder position in the artists conception). The Kontes glass sphere is shown on the left atop a laboratory beaker. 
The operational concept is shown in Figure 2. Note that as the cylinder is lowered in the water, the water pressure on the ram serves to "cock" or position the piston near the rupture disk. When the disk fails, the water pressure on the relatively large area of the piston results in a force much greater than that on the small area of the ram opposing it. The net force drives the piston (and ram) downward.



Figure 2) Conceptual view of the spherecracker piston assembly. The green rupture disk keeps the cylinder volume above the piston at nominal atmospheric pressure (left). An $\mathrm{O}$ ring seal at the cylinder opening for the ram keeps the cylinder chamber below the piston at nominal atmospheric pressure. When the disk fails (right) high pressure seawater rushes into the cylinder, driving the piston and ram downwards. 


\section{Field trials}

Field tests of the spherecracker assembly were initially conducted on Benthos Inc. deep sea glass flotation spheres [5]. The spheres consist of two identical hemispheres sealed together by a gasket and in a partial vacuum. These spheres have a $30.5 \mathrm{~cm}$ (12 inch) inner diameter, a $1.27 \mathrm{~cm}(0.5$ inch) wall thickness, and a depth rating of 11,000 meters. The depth rating of these spheres assured their survival at nominal SOFAR channel depths of $500-1200$ meters. The challenge was to cause sphere failure and implosion at these depths. A water reservoir was attached to the spherecracker pressure disc chamber and hydraulically pressurized until the disk failed at the nominal pressure of $1000 \mathrm{psi}$. The sphere was otherwise in ambient lab conditions with no significant pressure differential between the outside and inside of the sphere. The piston ram was driven into the sphere with sufficient force to cause the sphere to shatter into several large fragments. After the successful lab test, three tests were conducted at sea, all with rupture disks calibrated to fail at 1000 psi.

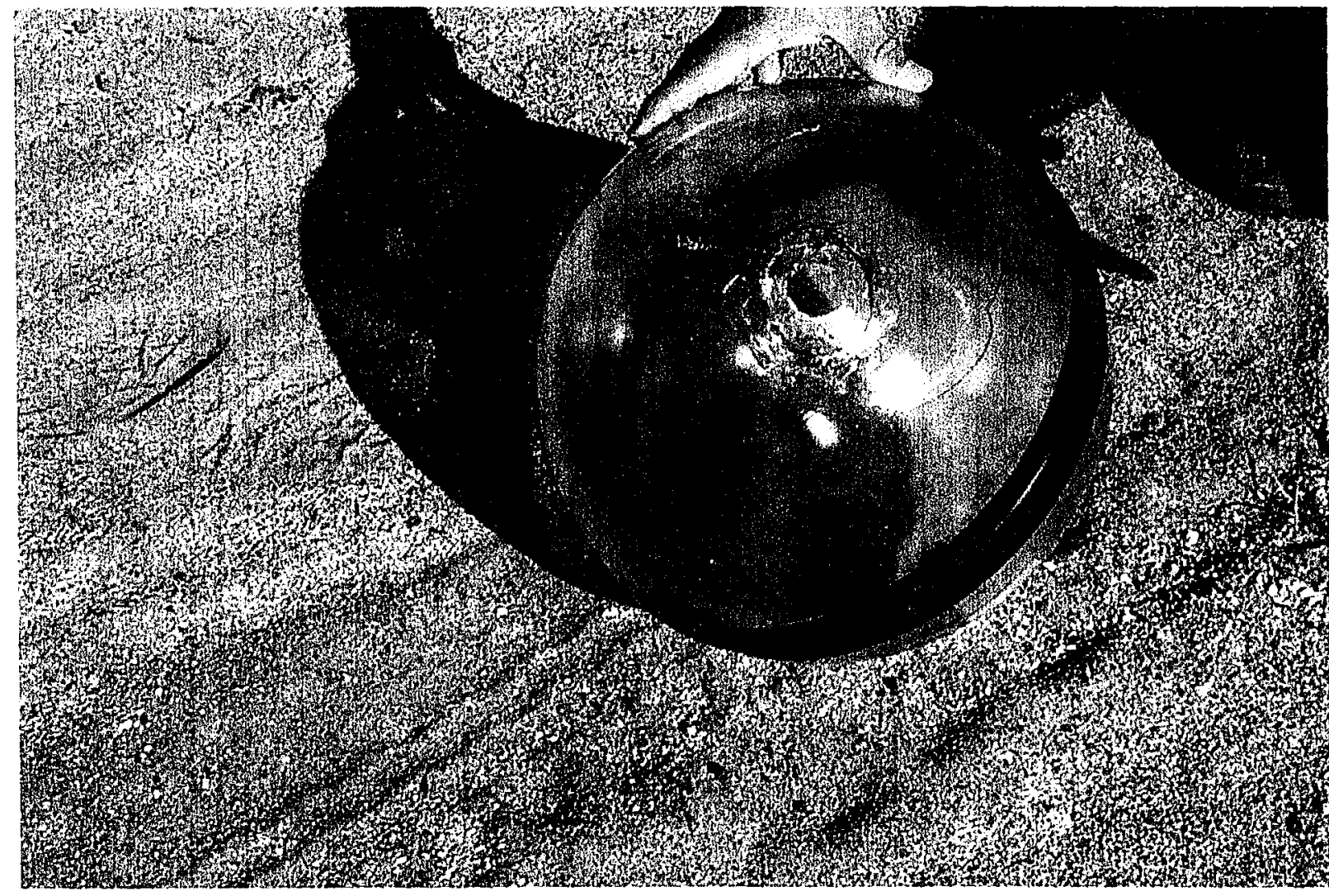

Figure 3) The remains of the Benthos sphere after the spherecracker successfully punched a hole at $\mathbf{7 0 0}$ meters nominal depth. Catastrophic failure and implosion was not initiated.

The spherecracker mechanism successfully punched a hole in the Benthos sphere at the nominal pressure of $1000 \mathrm{psi}$ in each test. However, despite the violent inrush of high pressure water through the hole and into the low pressure interior, the spheres did not otherwise fail. The ram was positioned to punch a hole at different parts of the sphere in each test (hemisphere pole, mid-lati- 
tude, and equator). The results were the same as shown in Figure 3. The external water pressure effectively strengthened the sphere enough to prevent crack propagation and catastrophic failure from the punched hole. We concluded that the Benthos spheres were too thick-walled to be used as an imploding source at nominal SOFAR channel depths.

A different type of glass sphere was also tested during the Benthos sphere tests. These spheres were made by the Custom Glass Shop, a Division of Kontes Glass Company [6]. The spheres are a special order modification of a standard 22-liter laboratory boiling flask. The wall thickness is nominally $0.64 \mathrm{~cm}(0.25$ inches $)$ but, since each sphere is hand blown, the wall thickness can vary considerably. Our concern with these spheres was their survival at nominal SOFAR axis depths. Initial tests were conducted without the spherecracker. The first test successfully lowered a sphere to 183 meters depth without self-failure. Failure was subsequently initiated at $150 \mathrm{~m}$ depth with a falling pipe and a large implosion signal was recorded.

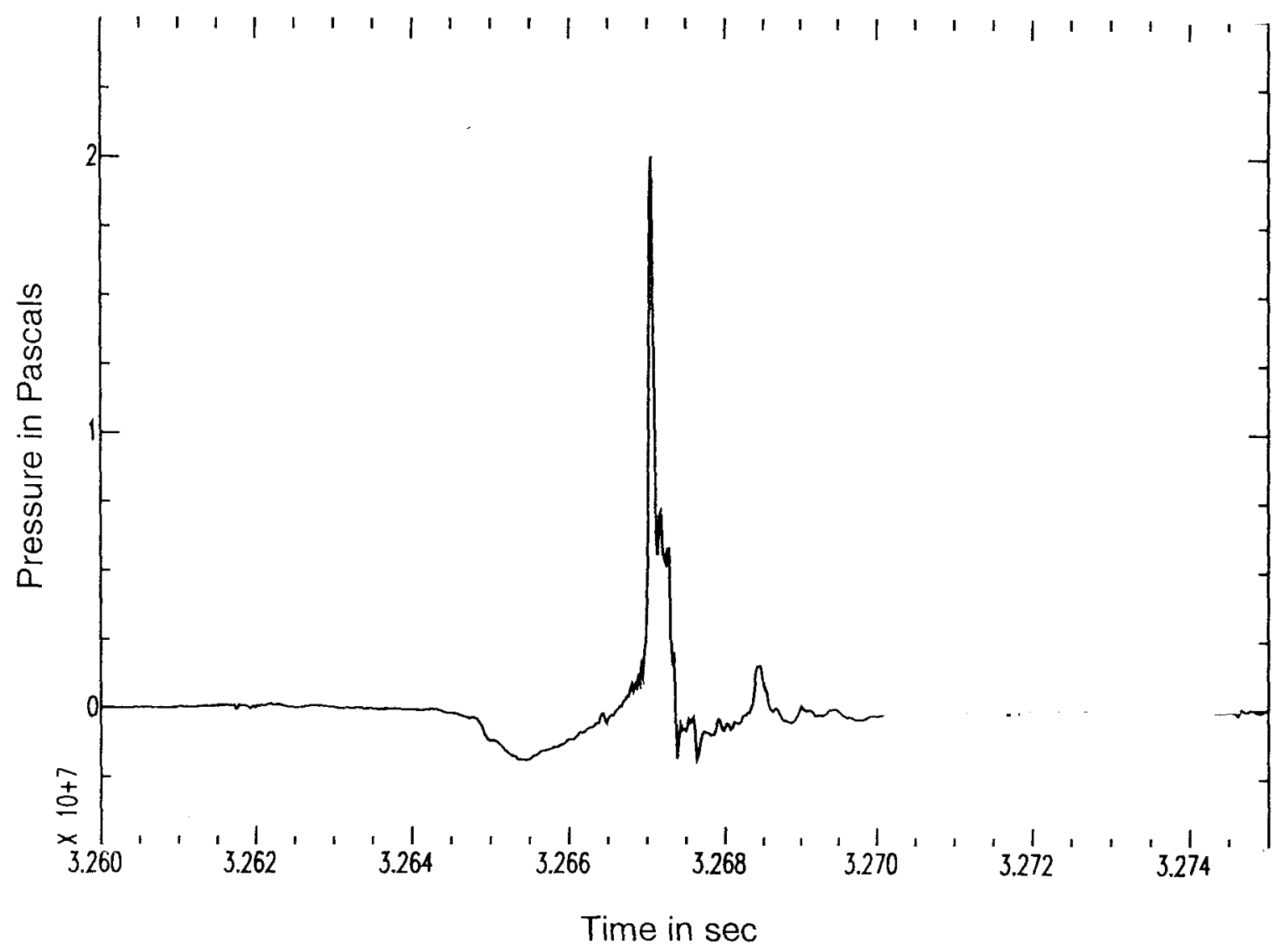

Figure 4) The calibrated pressure amplitude of the implosion in Pascals as recorded by the suspended hydrophone and projected back to 1 meter.

The second test, conducted in deep water, lowered the sphere to failure depth without the spherecracker. This occurred at about 1600 meters depth. The CGS sphere and the spherecracker mechanism appeared to be the right combination for SOFAR depths. 
An opportunity to conduct a field test with the CGS sphere and spherecracker came in Feb., 2000. The spherecracker was loaded aboard the R.V. Sproul and tested west of San Diego at water depths around 1200 meters. The spherecracker was loaded with a 22 liter CGS glass sphere and was lowered until a 1000 psi rupture disk failed. After initiating a successful implosion, the spherecracker was recovered undamaged. By reloading the device with a new pressure disk and glass sphere, it could be used repeatedly.

The impulsive hydroacoustic signal recorded from the implosion allowed accurate determinations of hydrophone depth, implosion depth and water depth from the surface reflected, bottom reflected, and 2 nd bottom reflected phases, respectively. The implosion occurred at 685 meters depth. The implosion signal was felt on the ship and recorded by a calibrated hydrophone suspended 13 meters deep. The recorded signal shown in Figure 4 was of short duration (less than 5 $\mathrm{msec}$ ) and with a small bubble pulse about $2 \mathrm{msec}$ after the main collapse. The event starts with a rarefaction as the sphere shatters and collapses. The large compressional pressure spike following the rarefaction occurs when the collapse reaches a minimum volume and reverses (begins expanding). The waveform was projected back to 1 meter by multiplying the hydrophone output by the distance of the hydrophone from the implosion, 672 meters. The peak pressure from the implosion is slightly larger than that from $1 \mathrm{lb}$ of TNT [7] at the depth of the test.

The amplitude spectrum of the implosion is shown in Figure 5. The source does not show any appreciable acoustic energy above background levels for frequencies below about $50 \mathrm{~Hz}$. Above $50 \mathrm{~Hz}$ the source amplitude rises to a broad maximum at frequencies between 200 and $800 \mathrm{~Hz}$. There is a relatively shallow high frequency roll-off of the source energy between $1 \mathrm{kHz}$ and 10 $\mathrm{kHz}$ whereas the noise rolls off more steeply in this band. The consequence is that the signal-tonoise ratio is highest at the highest frequency shown in the spectra: $10 \mathrm{kHz}$.

\section{Potential Utility as a Calibration Source}

Long range propagation of signals from small ( $1-2 \mathrm{lb})$ explosions within the SOFAR has been amply demonstrated [7]. Since the imploding sphere source within the SOFAR has a peak pressure amplitude similar to that of a small $(1 \mathrm{lb}$ ) explosion and similar frequency content, is reasonable to assume that the imploding sphere source signal will propagate for long distances within the SOFAR. The pressure amplitude spectra of the imploding sphere source shows that the bulk of the acoustic energy is well above the frequency band that is used for nuclear explosion monitoring (nominally $1-80 \mathrm{~Hz}$ ). This means that traveltimes, shadowing, diffraction, reflections, etc. would be calibrated at frequencies starting at the high end of the nuclear explosion monitoring band and extending beyond it. For some parameters (e.g. traveltime) this may be acceptable but for others (e.g. shadowing) it may lead to inaccuracies. Potential methods do exist to excite lower frequencies from the imploding sphere. They are: increase the volume of the sphere, implode the sphere at a shallower depth, and/or use multiple spheres simultaneously imploded. A larger sphere could be developed but it will be a relatively costly special order. A shallower implosion depth is simple to test. It will still need to be within the upper reaches of the SOFAR and will result in a smaller peak pressure amplitude. Multiple simultaneous sphere implosion could be easily tested using a single spherecracker and a bundle of spheres surrounding the mechanism and relying on shockwave-induced-implosion once the spherecracker implodes the central one. 


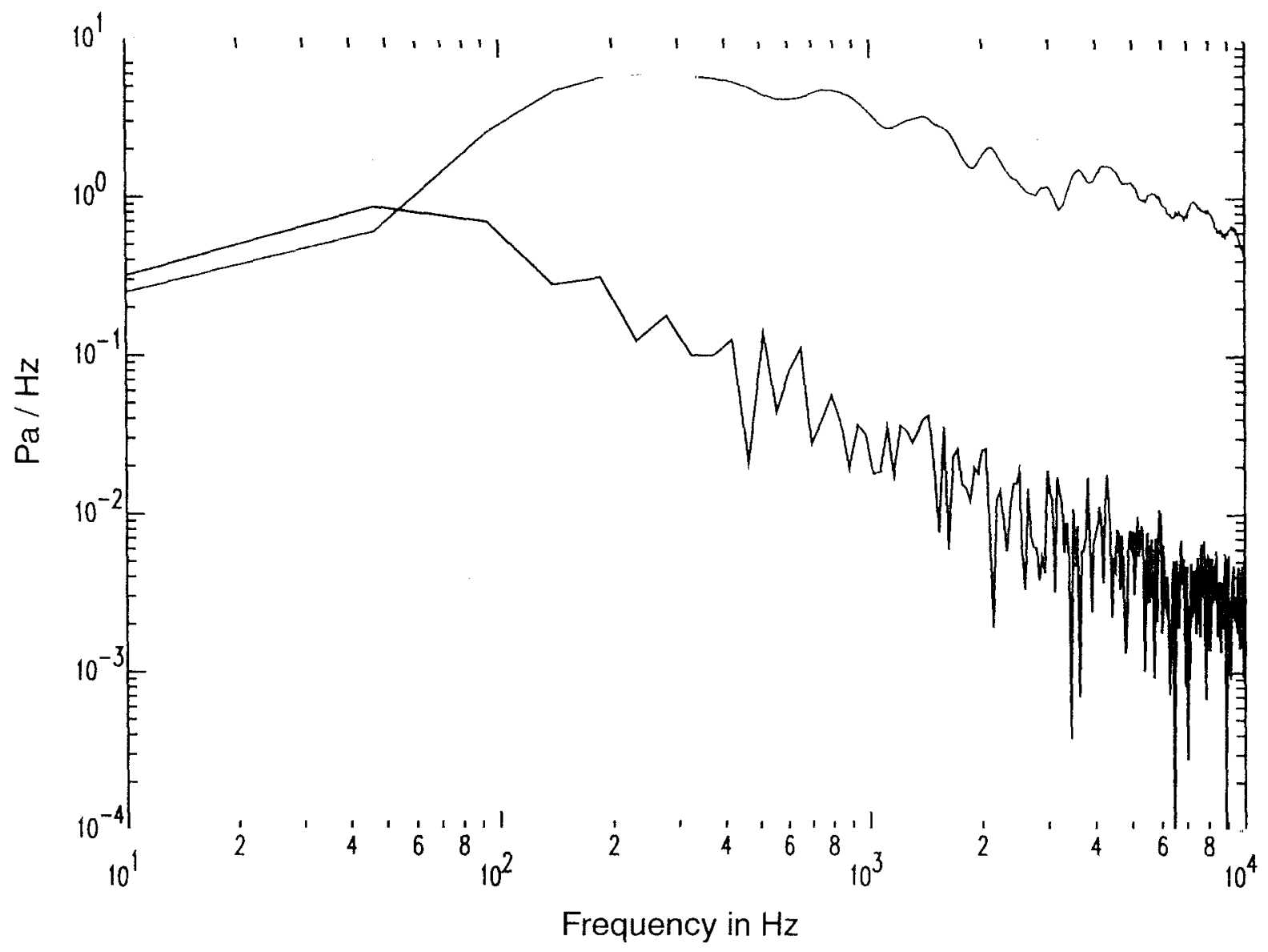

Figure 5) The implosion signal amplitude spectra (red) compared to pre-event noise (blue).

\section{Conclusions}

We have demonstrated that the spherecracker assembly can be made to work as an acoustic source at mid-ocean depths. The reliability of this source has not been established in the few tests conducted to date. Furthermore, although comparable explosions at the same depth can be detected at long ranges, provided the source and receiver are near the SOFAR axis and the SOFAR channel is not interrupted in the path connecting the two, actual experiments to verify this have yet to be done. The relatively high frequency content of the implosion source is a concern since it may limit the utility of some calibration measurements. A shallower implosion depth and/or a larger implosion source would shift the imploding sphere source spectra to lower frequencies and may mitigate that concern. Source phenomena modeling and further field testing is needed to determine the full range of hydroacoustic station calibration applications. 


\section{References}

[1] Informal Workshop on Hydroacoustics: Programme and Abstracts, Sponsored by CTBTO/ PTS and the DASE, Papeete, Tahiti, French Polynesia, Sept. 27 - Oct. 1, 1999.

[2] Isaacs, J.D. and A.E. Maxwell, The Ball-breaker; a Deep Water Signalling Device, J. Marine Res. 11,63-68, 1952.

[3] Orr, M. and M. Schoenberg, Acoustic Signatures from Deep Water Implosions of Spherical Cavities, JASA 59, No. 5, 1155-1159, 1976.

[4] Sauter, A., L. Dorman, E. Canuteson, Development of a Seismo/Acoustic Implosive Source, Fall AGU meeting, San Francisco, 1996 (abstract). Or see http://www.mpl.ucsd.edu/obs/reports/ ABM/implosive.agu.html.

[5] Benthos Inc., 49 Edgerton Dr., North Falmouth, MA, (800) 446-1222.

[6] CGS, a Division of Kontes Glass Co., 1022 Spruce St., Vineland, NJ, (800) 682-6644.

[7] Urick, R.J., Principles of Underwater Sound, Peninsula Publishing, Los Altos, CA., Copyright 1983.

\section{Acknowledgments}

We are indebted to Donna Blackman and Tim Minshull for support in some early field trials. Ted Farrell of BBN Inc. conducted one of the initial concept testing field experiments. Pat Lewis supported the lab test and some aspects of building the spherecracker. Jerry Sweeney provided useful criticism of the drafts. Support for the field test aboard the R/V Sproul was provided by the National Science Foundation under grant OCE 97-12605. 